\title{
Cognitive appraisals of dissociation in psychosis: a new brief measure
}

\author{
Emma Černis $^{1, *}$ (D), Jessica C. Bird ${ }^{1}$, Andrew Molodynski², Anke Ehlers ${ }^{2,3}$ (D) and Daniel Freeman ${ }^{1,2}$ \\ ${ }^{1}$ Oxford Cognitive Approaches to Psychosis, Department of Psychiatry, University of Oxford, Oxford, UK, ${ }^{2}$ Oxford Health \\ NHS Foundation Trust, Oxford, UK and ${ }^{3}$ Oxford Centre for Anxiety Disorders and Trauma, Department of Experimental \\ Psychology, University of Oxford, Oxford, UK \\ ${ }^{*}$ Corresponding author. Email: emma.cernis@psych.ox.ac.uk
}

(Received 29 June 2020; revised 17 September 2020; accepted 20 November 2020; first published online 28 December 2020)

\begin{abstract}
Background: Catastrophic cognitive appraisals, similar to those in anxiety disorders, are implicated in depersonalisation, a form of dissociation. No scales exist to measure appraisals of dissociative experiences. Dissociation is common in psychosis. Misinterpretations of dissociative experiences may maintain psychotic symptoms. Therefore, assessing appraisals in this context may be valuable.

Aims: The primary aim was to develop a measure of key appraisals of dissociation in psychosis. Secondary aims were to test the relationship between appraisals and psychotic experiences (paranoia and hallucinations), and determine whether appraisals explain additional variance in psychotic symptoms above dissociative symptoms.

Method: Fifty items were generated from transcripts of interviews with patients. The measure was developed and psychometrically validated via factor analysis of data from 9902 general population participants and 1026 patients with psychosis. Convergent validity, test-re-test reliability, and internal reliability were assessed. Regression analyses tested relationships with psychotic symptoms.

Results: A 13-item single-factor measure was developed. Factor analysis indicated good model fit $\left[\chi^{2}(65)=247.173\right.$, comparative fit index $(\mathrm{CFI})=0.960$, root mean square error of approximation $($ RMSEA $)=0.052$ ]. The scale had good convergent validity with a rumination (non-clinical: $r=0.71$; clinical: $r=0.73)$ and dissociation measure $(r=0.81 ; r=0.80)$, high internal consistency $(\alpha=0.93$; $\alpha=0.93$ ), and excellent 1 -week test-re-test reliability [intraclass correlation (ICC) $=0.90$ ]. It explained variance in psychotic symptoms (paranoia: $36.4 \%$; hallucinations: $35.0 \%$ ), including additional variance compared with dissociation alone (paranoia: $5.3 \%$; hallucinations: $2.3 \%$ ).

Conclusions: The Cognitive Appraisals of Dissociation in Psychosis (CAD-P) measure is a psychometrically robust scale identifying appraisals of dissociative experiences in psychosis and is associated with the presence of psychotic experiences. It is likely to prove useful for clinical assessment and research.
\end{abstract}

Keywords: cognitive appraisals; dissociation; hallucinations; paranoia; psychosis

\section{Introduction}

Dissociation is characterised by experiences of disconnection or alteration, such as feeling detached from one's own thoughts and emotions, finding one's own reflection unfamiliar, or seeing the world as 'flat' or unreal (Kennedy et al., 2013). Increasingly, dissociative experiences are being recognised as important transdiagnostic phenomena, occurring in diagnoses other than post-traumatic stress disorder (PTSD) (Lyssenko et al., 2018), and as

\footnotetext{
(C) British Association for Behavioural and Cognitive Psychotherapies 2020. This is an Open Access article, distributed under the terms of the Creative Commons Attribution licence (http://creativecommons.org/licenses/by/4.0/), which permits unrestricted re-use, distribution, and reproduction in any medium, provided the original work is properly cited.
} 
important clinical phenomena in their own right. For example, research is demonstrating how such experiences are independently associated with distress and increased self-harm (Černis et al., 2019) and suicidality (Calati et al., 2017). However, dissociation remains underrecognised by clinicians (Bailey and Brand, 2017).

Hunter and colleagues (2003) outline a cognitive model for the dissociative diagnosis of depersonalisation disorder (DPD), and Baker and colleagues (Baker et al., 2007) propose a model for the dissociative experiences of 'depersonalisation and feelings of unreality'. Both models highlight the role of 'catastrophic attributions' for the dissociative experience as central to the occurrence of the disorder, in a manner similar to that found in anxiety disorders, and particularly panic disorder (Clark, 1999). As in panic disorder, Hunter and colleagues propose that the transient symptoms experienced in DPD are misinterpreted as indicative of impending catastrophe. In panic disorder, this may be heart palpitations being mistaken as a sign of imminent heart attack; whilst in DPD a feeling of detachment may be taken as a sign of becoming 'mad'. Cognitive appraisals of dissociative symptoms as dangerous therefore result in an increase in anxiety (further exacerbating the dissociative symptoms), and lead to behaviours intended to neutralise the threat, but which actually serve to heighten attention towards them - such as monitoring symptoms and increasing introspection (Hunter et al., 2003). In the course of empirically testing this model, Hunter et al. (2014) demonstrated that a DPD group were less likely than a non-clinical group to make normalising attributions when prompted to generate as many reasons in one minute why they might experience various DPD, anxiety and neutral symptoms. In a second task, the DPD group were found to endorse catastrophic appraisals about mental illness or brain dysfunction in a manner similar to an anxiety disorder (obsessive compulsive disorder or panic disorder) comparison group. Both these findings support the hypothesis that anxiety-like cognitive appraisals may be important in this form of dissociation: however, they do not identify cognitive appraisals that are specific to dissociative experiences.

Dissociation may be particularly common in psychosis (Renard et al., 2017). Based on patient reports, we have characterised dissociation in this context as taking the form of an unanticipated subjective experience of anomaly (felt sense of anomaly, FSA), which may include experiences of disconnection, unfamiliarity and unreality (Černis et al., 2020). Consideration of dissociation may be important in psychosis research given the 'robust and well-replicated' associations between dissociation and psychotic symptoms (Longden et al., 2020), and suggestions that anomalous experiences - such as dissociation - may contribute to the development and maintenance of psychotic symptoms (Freeman, 2016; Garety et al., 2001). Consistent with the depersonalisation literature, anxiety processes have also been implicated in dissociation in this context (Černis et al., 2014; Freeman et al., 2013). However, to date, there has been no research into the cognitive appraisals that may be important in the maintenance of dissociation.

Clinicians can elicit in assessment appraisals of dissociation, but this process can be greatly aided by a questionnaire of key appraisals tested in large populations. It is also the case that before research can investigate the role of cognitive appraisals, a method for eliciting and measuring them is required. The objective of this study, therefore, was to develop a measure of key cognitive appraisals of dissociative experiences arising in the context of psychosis to aid clinicians and researchers in the detection, assessment and treatment of dissociation.

\section{Part 1: Development of the measure with a general population sample \\ Method}

Procedure and participants

The design was an online cross-sectional self-report questionnaire study. Participants were recruited via social media, the majority via Facebook advertisements. The advertisements were 
titled 'Understanding Dissociative Experiences' and stated that researchers were seeking participants to 'to complete questionnaires about different kinds of thoughts', and that they need not have experienced dissociation to take part. The information sheet described dissociation as a range of experiences "where people describe feeling "strange" or "disconnected" - they might feel like they are "spacing out", feel "unreal" or feel emotionally detached from the world'. Inclusion criteria were deliberately broad: any adult (age 18 years or over), usually resident in the United Kingdom (UK). There were no exclusion criteria.

All responses were collected via online surveys using Qualtrics (2018). The survey landing page contained the participant information sheet and statements regarding informed consent (British Psychological Society, 2017). Surveys were accessible on desktop and mobile web browsers. Incomplete surveys were retrieved automatically after a week of non-activity and added to the dataset.

There were three phases of data collection. Data collection for Phase 1 (full item pool) ran from 11 to 21 January 2019; and for Phase 2 (refined item pool) from 30 January to 26 February 2019. Phase 3 (test-re-test) data were collected from a subsample of Phase 2 participants between 5 and 23 April 2019. Following the removal of cases with high levels of missing data in the item pool (greater than $20 \%$ missing), sample sizes for the three phases were $n=1615, n=8287$ and $n=140$, respectively. For all samples, the majority of participants were White $(>94.00 \%)$ and female $(\geq 80.00 \%)$. The mean ages of the samples were $49.94(S D=14.40), 45.84(S D=14.86)$ and 46.69 years $(S D=15.27)$, respectively. See Table 1 for full demographic details.

\section{Measures}

Fifty items about cognitive appraisals of dissociation (e.g. 'this might last forever') were developed by the lead author from transcripts of interviews carried out for a qualitative study (Černis et al., 2020). In this qualitative study, 12 NHS patients with psychosis diagnoses and experience of dissociation were asked directly what thoughts they had in response to dissociation. Nine of the 12 patients scored within the range expected for dissociative disorders on the Dissociative Experiences Scale (Carlson and Putnam, 1993), with the mean score of $36.91(S D=20.00)$ indicating high levels of currently experienced dissociation within the group (Černis et al., 2020).

Participants in the online study rated the 50 items (see Supplementary material) for the past two weeks on a Likert scale from '0, never' to '4, always' with the instruction 'please rate how often you think the following when you are feeling strange, disconnected, unreal or "dissociated". Participants were prompted to answer to the best of their ability, and only to rate sensations that they believed were not caused by a physical health condition (e.g. migraine).

As well as a refined selection of the new items, participants in Phase 2 also completed two further measures, as follows.

Perseverative Thinking Questionnaire (PTQ; Ehring et al., 2011). This measure was included to enable the assessment of the new measure's convergent validity. Without an existing measure of cognitive appraisals of dissociation available for comparison, a measure of proneness to more general ruminative negative thinking was judged to be an appropriate alternative. Indeed, Hunter et al. (2003) implicate 'compulsive self-scrutiny' (p. 1459) in the maintenance of depersonalisation, suggesting a negative and ruminative quality to the cognitive processes involved in this form of dissociation.

The PTQ is a 15-item scale assessing trait ruminative negative thinking, such as 'I keep thinking about the same issues all the time'. Items are rated from ' 0 , never' to ' 4 , almost always' and refer to how the respondent 'typically think[s] about negative experiences or problems'. The range of this scale is 0 to 60 . Higher scores indicate greater severity of trait negative ruminative thinking.

In the Phase 2 group $(n=8287)$, the Cronbach's alpha for the PTQ was 0.97 and model fit for a one-factor 15 item scale structure was adequate for the large sample size $\left[\chi^{2}=8099.36\right.$, d.f. $=90$, $p<0.001$, comparative fit index $(\mathrm{CFI})=0.907$, Tucker-Lewis index (TLI) $=0.892$, root mean 
Table 1. Demographic data and descriptive statistics for all groups

\begin{tabular}{|c|c|c|c|c|c|}
\hline Participant group & & Phase 1 & Phase 2 & Test-re-test (subset of Phase 2) & Clinical \\
\hline $\mathbf{N}$ & & 1615 & 8287 & 140 & 1026 \\
\hline $\begin{array}{l}\text { Age } \\
\quad \text { Mean (SD) }\end{array}$ & & $49.94(14.40)$ & $45.84(14.86)$ & $46.69(15.27)$ & $41.49(12.29)$ \\
\hline Gender & Female: & 1433 (88.73\%) & 7103 (85.71\%) & $112(80.00 \%)$ & 300 (29.24\%) \\
\hline$n(\%)$ & $\begin{array}{l}\text { Male: } \\
\text { Other: }\end{array}$ & $\begin{array}{l}142(8.79 \%) \\
35(2.17 \%)\end{array}$ & $\begin{array}{c}962(11.61 \%) \\
165(1.99 \%)\end{array}$ & $\begin{aligned} 20 & (14.29 \%) \\
6 & (4.29 \%)\end{aligned}$ & $\begin{array}{c}717(69.88 \%) \\
5(0.49 \%)\end{array}$ \\
\hline Ethnicity & White (any): & $1551(96.04 \%)$ & $7866(94.92 \%)$ & $133(95.00 \%)$ & $687(66.96 \%)$ \\
\hline$n(\%)$ & Mixed/multiple: & $25(1.55 \%)$ & $175(2.11 \%)$ & $3(2.14 \%)$ & $43(4.19 \%)$ \\
\hline & Asian (any): & $12(0.74 \%)$ & $80(0.97 \%)$ & $2(1.43 \%)$ & 98 (9.55\%) \\
\hline & Black (any): & $3(0.19 \%)$ & $20(0.24 \%)$ & $0(0 \%)$ & $175(17.06 \%)$ \\
\hline & Other: & $6(0.37 \%)$ & $38(0.46 \%)$ & $0(0 \%)$ & $17(1.66 \%)$ \\
\hline $\begin{array}{l}\text { Measure } \\
\text { Mean (SD) }\end{array}$ & CAD-P & $19.05^{\star}(10.70)$ & $16.58(11.79)$ & $\begin{array}{l}\text { Week 1: } 20.12(10.69) \\
\text { Week 2: } 19.87(11.17)\end{array}$ & $18.70(13.35)$ \\
\hline & ČEFSA & - & $41.76(26.14)$ & - & $39.65(30.51)$ \\
\hline & PTQ & - & $34.97(13.83)$ & - & $30.32(16.38)$ \\
\hline $\begin{array}{l}\text { Have you ever experienced } \\
\text { mental health difficulties? } \\
n(\%)\end{array}$ & $\begin{array}{l}\text { Yes } \\
\text { No }\end{array}$ & $\begin{array}{l}1222(75.67 \%) \\
360(22.29 \%)\end{array}$ & $\begin{array}{l}6584(79.45 \%) \\
1557(18.79 \%)\end{array}$ & - & - \\
\hline $\begin{array}{l}\text { If 'yes', are these ongoing? } \\
n(\%)\end{array}$ & $\begin{array}{l}\text { Yes } \\
\text { No }\end{array}$ & $\begin{array}{l}886(72.50 \%) \\
312(25.53 \%)\end{array}$ & $\begin{array}{l}4919(74.71 \%) \\
1520(23.09 \%)\end{array}$ & - & - \\
\hline Diagnosis $n(\%)$ & $\begin{array}{l}\text { Schizophrenia } \\
\text { Schizoaffective } \\
\text { Delusional disorder } \\
\text { Psychotic disorder NOS** } \\
\text { First episode psychosis } \\
\text { Other SSD }\end{array}$ & - & - & - & $\begin{array}{c}662(64.52 \%) \\
153(14.91 \%) \\
14(1.36 \%) \\
69(6.73 \%) \\
105(10.23 \%) \\
23(2.24 \%)\end{array}$ \\
\hline
\end{tabular}

CAD-P Cognitive Appraisals of Dissociation in Psychosis

ČEFSA Černis Felt Sense of Anomaly scale

PTQ Perseverative Thinking Questionnair

SSD Schizophrenia Spectrum Disorder

*Contains item 'I'm so lonely' which was later amended to 'I am all alone'

${ }^{\star \star}$ Not otherwise specified (NOS) also includes unspecified non-organic psychosis 
square error of approximation $(\mathrm{RMSEA})=0.106$, standardised root mean square residual $(\mathrm{SRMR})=0.037]$.

Černis Felt Sense of Anomaly (ČEFSA) scale (Černis et al., submitted). The ČEFSA is a 35-item scale assessing dissociative experiences taking the form of subjective experiences of strangeness (FSA). This includes items such as 'I don't fully experience emotions' and 'I feel like a stranger to myself rated on a 5-point Likert scale from '0, never' to ' 4 always'. The range of this scale is 0 to 140 and rates the past 2 weeks. Higher scores indicate greater levels of FSA-type dissociative experiences.

Model fit in the Phase 2 group for this scale's 7-factor 35-item structure was good $\left(\chi^{2}=14831.29, \quad\right.$ d.f. $=539, \quad p<0.001, \quad$ CFI $=0.920, \quad$ TLI $=0.911, \quad$ RMSEA $=0.057$, SRMR $=0.048)$, with a high Cronbach's alpha (0.97).

\section{Statistical analysis}

Analyses were conducted in R, version 3.5.1 (R Core Team, 2018) with packages psych (version 1.8.12; Revelle, 2018) and lavaan (version 0.6-3.1295; Rosseel, 2018). Exploratory factor analyses (EFA) with oblimin rotation and maximum likelihood estimator were carried out to assess the structure of items and refine the item pool by discarding poor-fitting items. Items were judged to fit poorly if they loaded weakly to a factor (less than 0.3 ), had commonalities less than 0.3 , or loaded with similar strength onto multiple factors (loadings within 0.2). Note this latter criterion only applied during Phase 1 , as Phase 2 analysis indicated a one-factor structure. The number of factors to extract was determined through parallel analysis and inspection of the scree plot.

The final measure model was assessed using confirmatory factor analysis (CFA) with MLR robust maximum likelihood estimator. The measure's psychometric properties were also assessed, including a test-re-test reliability statistic, which used the test-re-test phase data collected specifically for this purpose. Convergent validity with the ČEFSA and PTQ were also assessed via Pearson's correlation, and internal reliability using Cronbach's alpha. Test-re-test reliability was examined using the intraclass correlation (ICC) between Week 1 and Week 2 data collected specifically for this purpose (test-re-test group).

\section{Results}

Measure development

Phase 1. Confirming that factor analysis was appropriate for the general population group, Bartlett's test of sphericity was significant $\left(\chi^{2}=51,166.67\right.$, d.f. $\left.=1225, p<0.001\right)$ and the Kaiser-Meyer-Olkin test of sampling adequacy was high $(\mathrm{KMO}=0.97)$.

Following the criteria for removing poor-fitting items, and after removing items with inconsistent theoretical content (e.g. appraisals referring to oneself, rather than to dissociative experiences), EFA of Phase 1 data $(n=1615)$ led to 27 of the original 50 items being discarded. Additionally, two items were re-worded at this stage to improve clarity of meaning (shown in Supplementary material): 'I'm so lonely' became 'I am all alone', and 'This must mean I'm not human' was re-worded to 'This must mean I'm an alien, ghost, or not human'.

Phase 2. In Phase 2, a new sample of general population respondents $(n=8287)$ completed the refined pool of 23 items. Confirming that factor analysis was appropriate, Bartlett's test of sphericity was significant $\left(\chi^{2}=12,3890.00\right.$, d.f. $\left.=253, p<0.001\right)$ and the Kaiser-Meyer-Olkin test of sampling adequacy was high $(\mathrm{KMO}=0.97)$. This sample was split using R's random sampling function into a development $(n=4143)$ and validation $(n=4144)$ sample for analysis.

In the development sample, the scree plot and parallel analysis tests indicated that a one-factor structure was the most appropriate fit for the data. Following factor analysis, a further six items 
Table 2. Factor loadings for the final items of the measure for the non-clinical and clinical validation groups

\begin{tabular}{|c|c|c|c|}
\hline \multirow[b]{2}{*}{ Item } & & \multicolumn{2}{|l|}{ Participant group } \\
\hline & & $\begin{array}{c}\text { Non-clinical (Phase 2, validation } \\
\text { sample) }(n=4144)\end{array}$ & $\begin{array}{c}\text { Clinical } \\
(n=1026)\end{array}$ \\
\hline 1 & I can't trust my own mind. & 0.78 & 0.69 \\
\hline 2 & Someone has done something to me. & 0.58 & 0.64 \\
\hline 3 & Something is terribly wrong. & 0.78 & 0.76 \\
\hline 4 & I'm losing my mind. & 0.82 & 0.78 \\
\hline 5 & I'm not really 'me'. & 0.76 & 0.77 \\
\hline 6 & I am all alone. & 0.71 & 0.66 \\
\hline 7 & I don't look right to other people right now. & 0.75 & 0.71 \\
\hline 8 & I must be sick. & 0.76 & 0.70 \\
\hline 9 & I'm not in the same world as everyone else. & 0.65 & 0.73 \\
\hline 10 & This is because I am evil. & 0.55 & 0.58 \\
\hline 11 & Now I won't be able to do the things I wanted. & 0.72 & 0.68 \\
\hline 12 & It's not me in control right now. & 0.73 & 0.74 \\
\hline 13 & This might last forever. & 0.73 & 0.72 \\
\hline
\end{tabular}

were discarded for poor fit, and four items due to high correlated residuals with other items. The resulting 13 -item one-factor model explained $52 \%$ of the variance.

CFA in the validation sample demonstrated a good fit for this model $\left(\chi^{2}=900.639\right.$, d.f. $=65$, $p<0.001, \mathrm{CFI}=0.962$, TLI $=0.955, \mathrm{RMSEA}=0.056, \mathrm{SRMR}=0.025)$. Factor loadings of the final items in this sample are shown in Table 2.

\section{Measure reliability and validity}

The 13-item Cognitive Appraisals of Dissociation in Psychosis (CAD-P) measure had excellent internal consistency $(n=4144$, Cronbach's $\alpha=0.93)$. It was also found to have excellent oneweek test-re-test reliability $[n=140, \quad$ ICC $=0.90,95 \%$ confidence interval $(\mathrm{CI})=0.85-$ $0.94, p<0.001]$.

Furthermore, good convergent validity was found when tested by Pearson's correlation with the PTQ (Ehring et al., 2011) $(n=8287, r=0.71, p<0.001)$, and with the ČEFSA (Černis et al., submitted) $(n=8287, r=0.81, p<0.001)$. As there was a significant difference between genders on the PTQ $(p=0.023$; females: mean $=34.97, S D=13.85$; males: mean $=33.85$, $S D=13.96)$, convergent validity was also tested for females and males separately. Both indicated high correlation between the CAD-P and PTQ (females: $n=7103, r=0.71$, $p<0.001$; males: $n=962, r=0.72, p<0.001$ ).

\section{Part 2: Clinical validation}

\section{Method}

\section{Procedure and participants}

The design was a cross-sectional self-report questionnaire study. The study was supported by the National Institute of Health Research (NIHR) Clinical Research Network (CRN). Participants were recruited by CRN research assistants and clinical studies officers embedded in clinical teams and Research and Development departments across 36 NHS trusts. Inclusion criteria were broad: any person (age 16 years or over), currently under the care of an NHS mental health service, with a diagnosis of non-affective psychosis, who was willing and able to give informed consent to participate. Exclusion criteria were: insufficient English language to complete the questionnaires with support, and an affective psychosis diagnosis (i.e. psychotic depression, bipolar disorder). 
Recruitment took place between 18 October 2019 and 19 March 2020. Datasets from 1038 participants were returned. For this analysis, only cases without high levels of missing data in the CAD-P measure (less than or equal to $20 \%$ missing) were retained for analysis. This resulted in a participant group of 1026 patients.

In this group, the majority of participants were White $(66.96 \%)$, male $(69.88 \%)$ and had a diagnosis of schizophrenia (64.52\%). The mean age of the sample was 41.49 years $(S D=12.29)$. See Table 1 for full demographic and descriptive details.

For the second part of the analysis, only cases without high levels of missing data (less than or equal to $20 \%$ missing) on the CAD-P, ČEFSA and the two psychosis symptom measures were retained. This resulted in a subgroup of $n=1015$. The demographic details and descriptive statistics for this subgroup can be found in the Supplementary material.

\section{Measures}

Participants answered the 13-item version of the CAD-P developed in Part 1 (see Appendix for the full scale), as well as the Perseverative Thinking Questionnaire (Ehring et al., 2011) and the Černis Felt Sense of Anomaly (ČEFSA) scale (Černis et al., submitted), as described in Part 1. In this group $(n=1026)$ both scales had good internal reliability (ČEFSA: Cronbach's alpha $=0.97$; PTQ: 0.96 ) and good model fit (ČEFSA: $\chi^{2}=1530.378$, d.f. $=539, p<0.001$, CFI $=0.932$, $\mathrm{TLI}=0.925, \quad \mathrm{RMSEA}=0.042, \quad \mathrm{SRMR}=0.042 ; \quad$ PTQ: $\quad \chi^{2}=450.929, \quad$ d.f. $=90, \quad p<0.001$, $\mathrm{CFI}=0.949, \quad \mathrm{TLI}=0.941, \quad \mathrm{RMSEA}=0.063, \quad \mathrm{SRMR}=0.029)$. Participants also completed measures of paranoia and hallucinations, as follows.

Revised Green Paranoid Thoughts Scale (R-GPTS Persecution; Freeman et al., 2019). The R-GPTS is a scale assessing paranoia via ideas of reference and persecution subscales. The persecution subscale of the R-GPTS was used in this study. This subscale consists of ten items (e.g. 'Certain individuals have had it in for me'), rated over the past month on a 5-point Likert scale from ' 0 , not at all' to '4, totally'. Higher scores indicate higher levels of paranoia, with a score above 18 indicating 'severe' levels of perceived persecution.

In the clinical group $(n=1026)$, the Cronbach's alpha for this scale was good $(0.92)$, as was the model fit $\left(\chi^{2}=4007.887\right.$, d.f. $=45, p<0.001, \mathrm{CFI}=0.968, \mathrm{TLI}=0.959, \mathrm{RMSEA}=0.060$, $\operatorname{SRMR}=0.025)$.

Specific Psychotic Experiences Questionnaire (SPEQ-H; Ronald et al., 2013). The SPEQ consists of four scales which each assess a key psychotic experience. The hallucinations scale (SPEQ-H) was used in this study. This asks respondents to rate how frequently they have recently had particular experiences (e.g. 'How often do you: hear noises or sounds when there is nothing about to explain them?') using a 6-point Likert scale (' 0 , not at all' to ' 5 , daily'). Higher scores indicate higher levels of hallucinatory experiences. This scale was adapted to include two further items assessing voice hearing: 'How often do you... hear voices saying words or sentences when there is no one around that might account for it' and ' ... hear two or more unexplained voices talking to each other'. Indicating that the additional items are consistent with the original items, the Cronbach's alpha for the adapted SPEQ-H in this group $(n=1026)$ was high (0.93). Model fit for a single-factor scale structure was also adequate for a large sample size $\left(\chi^{2}=711.491, \quad\right.$ d.f. $=44, \quad p<0.001, \quad$ CFI $=0.831, \quad$ TLI $=0.789$, $\mathrm{RMSEA}=0.122, \mathrm{SRMR}=0.065)$.

\section{Statistical analysis}

Analyses were conducted in R, version 3.6.3 (R Core Team, 2020) with packages psych (version 1.9.12.31; Revelle, 2019) and lavaan (version 0.6-5; Rosseel et al., 2019). 


\section{Psychometric validation}

The single factor model identified in Part 1 was assessed using CFA with MLR robust maximum likelihood estimator in the clinical subsample $(n=1007)$. Convergent validity of the scale with the ČEFSA and PTQ were assessed via Pearson's correlation, and its internal reliability via Cronbach's alpha. Test-re-test data were not collected for this population.

\section{Relationship to psychosis variables}

Additionally, to begin to demonstrate the value of the CAD-P measure in psychosis research, linear regression models were used to determine whether cognitive appraisals of dissociation explained more variance in psychotic symptoms (paranoia and hallucinations) than dissociative experiences alone.

Data from the subgroup of 1015 participants with low $(\leq 20 \%)$ missing data on the CAD-P, ČEFSA, R-GPTS and SPEQ-H were used for this analysis. Missing data for all four scales were imputed using the mice package (version 3.8.0; van Buuren and Groothuis-Oudshoorn, 2020) in order to obtain total scores. Simple and multiple linear regression analyses were then carried out entering the ČEFSA and CAD-P measures as independent variables and the R-GPTS and SPEQ-H measures as dependent variables. As well as inspecting and comparing the estimates of each regression model, models were also formally tested for significant difference using ANOVA analyses.

\section{Results}

\section{Psychometric validation}

CFA in the clinical group indicated a single factor model had a good fit to the data $\left(\chi^{2}=253.630\right.$, d.f. $=65, p<0.001$, CFI $=0.959$, TLI $=0.950$, RMSEA $=0.053$, SRMR $=0.029$ ). Factor loadings for the final items in this group are shown in Table 2.

In this sample, the CAD-P had good internal consistency (Cronbach's $\alpha=0.93$ ) and good convergent validity with the ČEFSA (Černis et al., submitted) $(r=0.80, p<0.001)$ and the PTQ (Ehring et al., 2011) $(r=0.73, p<0.001)$. As there was a significant difference between genders for the PTQ $(t=2.74, p=0.006$; females: mean $=32.57, S D=16.98$; males: mean $=29.36, S D=16.09)$, convergent validity between the PTQ and CAD-P was also confirmed for females and males separately (females: $r=0.70, \quad p<0.001$; males: $r=0.75, p<0.001)$.

\section{Relationship to psychosis variables}

Paranoia. With the R-GPTS Persecution scale as the dependent variable, simple regression analysis with the ČEFSA as the independent variable found that ČEFSA scores explained $34.3 \%$ of the variance in R-GPTS scores $(\beta=0.586,95 \% \mathrm{CI}: 0.566-0.606, p<0.001)$. On its own, CAD-P explained $36.4 \%$ of the variance in R-GPTS $(\beta=0.604$, 95\% CI: $0.559-$ $0.649, p<0.001)$.

When both ČEFSA and CAD-P were entered as independent variables, the multiple regression model explained $39.6 \%$ of the variance in R-GPTS scores (ČEFSA: $\beta=0.291,95 \%$ CI: $0.260-0.322$, $p<0.001$; CAD-P: $\beta=0.374,95 \%$ CI: $0.303-0.446, p<0.001)$ : an increase of $5.3 \%$ compared with ČEFSA alone. An ANOVA confirmed that this model was a significantly better fit to the data than either simple regression model $(F=53.72$, d.f. $=1, p<0.001)$.

Hallucinations. A simple linear regression model with SPEQ-H as the dependent variable and ČEFSA as the independent variable explained $40.1 \%$ of the variance in SPEQ-H scores $(\beta=0.634,95 \% \mathrm{CI}$ : $0.609-0.659, p<0.001)$. A simple linear regression model with CAD-P as the independent variable explained $35.0 \%$ of the variance $(\beta=0.593,95 \%$ CI: $0.532-0.653, p<0.001)$. 
Including both variables in a multiple regression model explained $42.4 \%$ of variance in SPEQ-H scores (ČEFSA: $\beta=0.441,95 \%$ CI: $0.401-0.481, p<0.001$; CAD-P: $\beta=0.245$, 95\% CI: $0.153-$ $0.337, p<0.001$ ): an increase of $2.3 \%$ compared with the simple regression model of ČEFSA alone. An ANOVA found that the multiple regression model was a statistically significantly better fit to the data than either simple regression model $(F=129.28$, d.f. $=1, p<0.001)$.

\section{Discussion}

To the authors' knowledge, this study - and its resulting measure - are the first to explicitly address cognitive appraisals of dissociative experiences in the context of psychosis. The measure identifies 13 key cognitive appraisals, all loading onto a single factor. A copy of the full scale can be found in the Appendix. The CAD-P scale has good psychometric properties in both non-clinical and clinical (psychosis) groups: the model-fit is robust, internal reliability is high, and convergent validity with related measures is also good. Where test-re-test reliability was assessed, this was also high for a 1-week interval. When added to regression models, the CAD-P explained additional variance in both paranoia and hallucinations scores in a clinical group, above and beyond that explained by a dissociation measure alone. In general, therefore, the novel scale appears to fulfil the study aim of providing a method for assessing cognitive appraisals relevant to dissociation in psychosis for clinical and research purposes.

Items of the CAD-P, such as 'I must be sick' and 'I'm losing my mind' are consistent with the proposed similarities between dissociation and anxiety appraisals (Baker et al., 2007; Hunter et al., 2003; Hunter et al., 2014). These appraisals reflect concerns about dissociative experiences being inherently dangerous, or a sign of imminent danger. However, this study also suggests that a number of other appraisals may be important to the experience of dissociation in psychosis. Items reflecting negative beliefs about self ('This is because I'm evil') and other people ('Someone has done something to me') reflect current understanding of beliefs common in patients experiencing high levels of paranoia (Collett et al., 2016; Stopa et al., 2013). Further, and of particular note, a number of items indicate concerns about control and ownership over oneself and one's actions ('I can't trust my own mind', 'I'm not really "me"' and 'It's not me in control right now'). Whilst maladaptive beliefs about control are a feature of a number of mental health disorders, these statements suggest that experiencing one's self and internal world as 'other' can directly lead people to doubt their autonomy and control. Following Maher (1974), who proposed that within a context of particular beliefs and cognitive biases, anomalous experiences 'demand explanation' which may culminate in the adoption of an explanatory delusional belief, it is understandable how the above appraisals might result in delusions of control or passive influence. It is interesting to note that these particular psychotic symptoms have been found to be more common in dissociative identity disorder than in psychosis patients (Laddis and Dell, 2012). These items of the CAD-P, therefore, appear to capture concerns that are specific to the experience of dissociation, but which may be highly relevant to the 'fuzzy' boundary between dissociation and psychosis (Renard et al., 2017). Further exploration of dissociation - and cognitive appraisals of such experiences may therefore be particularly appropriate in clinically high-risk (CHR) or prodromal presentations. The broad range of items might be a strength of the CAD-P: clinicians may wish to use the scale initially to enquire further about which appraisals cause the most distress or are the most relevant to their client's specific circumstances. Subsequent treatment could then be tailored to these appraisals, for example, through the use of behavioural experiments in therapies following a cognitive behavioural approach.

Another strength of the CAD-P is its good psychometric properties in the non-clinical and clinical groups. Regarding convergent validity, it is promising that the CAD-P correlated highly in both samples with the PTQ (Ehring et al., 2011). The PTQ measures ruminative 
negative thinking style, which may be expected to occur as a result of catastrophic cognitive appraisals. Rumination can also be seen as a form of intense internal focus, which Hunter and colleagues (Hunter et al., 2003) propose may be common in depersonalisation. The current study determined a correlational relationship between the cognitive appraisals of dissociation and rumination - however, our understanding of dissociation may benefit from research which seeks to determine direction of effect between the two constructs. The CAD-P may be a valuable tool for research such as this in studies using experimental designs, network analysis methods, and treatment outcome (mechanism) studies.

This utility was demonstrated in the current study by using the CAD-P to demonstrate that cognitive appraisals of dissociation explained additional variance in paranoia (persecution) and hallucinations over and above dissociation alone. Interestingly, there were differences between the results for hallucinations and paranoia. For paranoia, cognitive appraisals of dissociation explained approximately the same proportion of variance as FSA-dissociation. However, for hallucinations, cognitive appraisals of dissociation explained closer to half the proportion of variance as did FSA-dissociation. Tentatively, these findings could be interpreted as reflecting the robust relationship between dissociative experience and hallucinations (Longden et al., 2020), and the importance of cognition in the development of paranoia (Freeman, 2016). A clinically relevant aim for future research may therefore be to explore the impact on psychotic symptoms of cognitive restructuring for appraisals of dissociation; in particular, whether this may be a mechanism to lessen positive psychotic symptoms. Exploration of these appraisals in relation to negative psychotic symptoms would also be of interest, particularly as many of the CAD-P items contain negative beliefs, or describe passive responses to dissociative experiences.

Limitations of the current study include the manner in which items were developed. As item development drew upon only 12 clinical interviews, it is possible that important cognitions were not elicited and therefore not added to the item pool. For example, cognitive appraisals regarding brain damage or functional impairment have been demonstrated as important in PTSD patients (Samuelson et al., 2017), and may also be relevant to dissociation or psychosis.

The sampling method presents another potential limitation of the current study. Whilst providing large sample sizes - a strength of the study that allowed for rigorous analysis recruitment via Facebook advertisements in Part 1 resulted in a biased sample. The nonclinical samples were drawn from the general population of the UK only, and contained a very high proportion of female and White respondents, with notably high levels of selfreported ongoing mental health difficulties. It is likely that this is the result of self-selection bias due to the title and description of the study sample. Despite the large number of male respondents and respondents from a range of ethnic groups, the participant pool was therefore not representative of the general population. Due to the sampling method, participation was limited to those aged 18 or over. Further validation of the CAD-P in a younger age range would extend the utility of the scale. In particular, this is likely to be important in studies of participants with CHR presentations, where the age range might be expected to include people below the age of 18 .

Finally, there are two methodological limitations. Test-re-test data were not collected from the clinical participants in Part 2. As a result, despite the strong test-re-test result in the non-clinical sample (Part 1), this property of the scale remains unknown in clinical contexts and requires further validation. Convergent validity of the CAD-P was assessed using only one dissociation measure. Analysis of validity using a broader range of dissociative measures (particularly the Dissociative Experiences Scale; Carlson and Putnam, 1993) would also be beneficial.

Nonetheless, the CAD-P scale may prove to be a useful tool for researchers and clinicians to identify, assess and investigate cognitive appraisals in individuals with dissociation, especially in the context of psychosis. 
Supplementary material. To view supplementary material for this article, please visit: https://doi.org/10.1017/ S1352465820000958

Acknowledgements. The authors would like to thank the 12 participants interviewed in the qualitative study from which the item pool was developed for sharing their experiences and expertise. We would like to thank the R\&D and NIHR CRN staff within the many mental health trusts involved in the 'Exploring Unusual Feelings' study.

We would like to thank the following NHS trusts for participating: Avon and Wiltshire Mental Health Partnership NHS Trust; Black Country Healthcare NHS Foundation Trust; Barnet, Enfield \& Haringey Mental Health NHS Trust; Birmingham and Solihull Mental Health NHS Foundation Trust; Berkshire Healthcare NHS Foundation Trust; Birmingham Women's and Children's NHS Foundation Trust; Camden and Islington NHS Foundation Trust; Central and North West London NHS Foundation Trust; Coventry and Warwickshire Partnership NHS Trust; Cambridge and Peterborough NHS Foundation Trust; Cornwall Partnership NHS Foundation Trust; Cheshire and Wirral Partnership NHS Foundation Trust; Cumbria, Northumberland, Tyne and Wear NHS Foundation Trust; Dorset Healthcare University NHS Foundation Trust; Dudley and Walsall Mental Health Partnership NHS Trust; Devon Partnership NHS Trust; East London NHS Foundation Trust; Gloucestershire Health and Care NHS Foundation Trust; Hertfordshire Partnership University NHS Foundation Trust; Humber Teaching NHS Foundation Trust; Kent and Medway NHS and Social Care Partnership Trust; Leicestershire Partnership NHS Trust; Midlands Partnership NHS Foundation Trust; Mersey Care NHS Foundation Trust; North East London Foundation Trust; North Staffordshire Combined Healthcare NHS Trust; Oxford Health NHS Foundation Trust; Pennine Care NHS Foundation Trust; Surrey and Borders Partnership NHS Foundation Trust; Sheffield Health \& Social Care NHS Foundation Trust; Solent NHS Trust; Somerset Partnership NHS Foundation Trust; Southern Health NHS Foundation Trust; Tees, Esk and Wear Valleys NHS Foundation Trust; Worcestershire Health and Care NHS Trust; and West London NHS Trust.

Financial support. This study was funded by a Wellcome Trust Clinical Doctoral Fellowship awarded to E.Č. (102176/B/13/ Z). A.E. is funded by the Wellcome Trust (200796), the Oxford Health NIHR Biomedical Research Centre (BRC-1215-20005) and a NIHR Senior Fellowship. D.F. and J.B. are funded by an NIHR Research Professorship awarded to D.F. (RP-2014-05003). The views expressed are those of the authors and not necessarily those of the NHS, the NIHR or the Department of Health. The funders had no role in study design, data collection and analysis, decision to publish, or preparation of the manuscript.

\section{Conflicts of interest. None.}

Ethical statements. Authors abided by the Ethical Principles of Psychologists and Code of Conduct as set out by the BABCP and BPS. For Part 1 (non-clinical): the study received ethical approval from the Central University Research Ethics Committee of the University of Oxford (reference no. R61315/RE002). For Part 2 (clinical): the study received ethical approval from the NHS Health Research Authority, London (City \& East) Research Ethics Committee (reference no. 19/LO/1394).

\section{References}

Bailey, T. D., \& Brand, B. L. (2017). Traumatic dissociation: theory, research, and treatment. Clinical Psychology Science and Practice, 24, 170-185. doi: 10.1111/cpsp.12195

Baker, D., Hunter, E., Lawrence, E., David, A., Medford, N., \& Sierra, M. (2007). Overcoming Depersonalisation and Feelings of Unreality: A Self-Help Guide Using Cognitive Behavioural Techniques. Hachette UK.

British Psychological Society (2017). Ethics Guidelines for Internet-Mediated Research. INF206/04.2017. Leicester: BPS.

Calati, R., Bensassi, I., \& Courtet, P. (2017). The link between dissociation and both suicide attempts and non-suicidal selfinjury: meta-analyses. Psychiatry Research, 251, 103-114. doi: 10.1016/j.psychres.2017.01.035

Carlson, E. B., \& Putnam, F. W. (1993). An update on the Dissociative Experiences Scale. Dissociation, 6, 16-27.

Černis, E., Beierl, E., Molodynski, A., Ehlers, A., \& Freeman, D. (submitted). A new perspective and assessment measurement for common dissociative experiences: 'Felt Sense of Anomaly'.

Černis, E., Chan, C., \& Cooper, M. (2019). What is the relationship between dissociation and self-harming behaviour in adolescents? Clinical Psychology \& Psychotherapy, 26, 328-338. doi: 10.1002/cpp.2354

Černis, E., Dunn, G., Startup, H., Kingdon, D., Wingham, G., Pugh, K., Cordwell, J., Mander, H., \& Freeman, D. (2014). Depersonalization in patients with persecutory delusions. Journal of Nervous and Mental Disease, 202, $752-758$. doi: 10.1097/NMD.0000000000000185

Černis, E., Freeman, D., \& Ehlers, A. (2020). Describing the indescribable: a qualitative study of dissociative experiences in psychosis. PLoS One, 15, e0229091. doi: 10.1371/journal.pone.0229091

Clark, D. M. (1999). Anxiety disorders: why they persist and how to treat them. Behaviour Research and Therapy, 37, S5. doi: 10.1016/S0005-7967(99)00048-0 
Collett, N., Pugh, K., Waite, F., \& Freeman, D. (2016). Negative cognitions about the self in patients with persecutory delusions: an empirical study of self-compassion, self-stigma, schematic beliefs, self-esteem, fear of madness, and suicidal ideation. Psychiatry Research, 239, 79-84. doi: 10.1016/j.psychres.2016.02.043

Ehring, T., Zetsche, U., Weidacker, K., Wahl, K., Schönfeld, S., \& Ehlers, A. (2011). The Perseverative Thinking Questionnaire (PTQ): validation of a content-independent measure of repetitive negative thinking. Journal of Behavior Therapy and Experimental Psychiatry, 42, 225-232. doi: 10.1016/j.jbtep.2010.12.003

Freeman, D. (2016). Persecutory delusions: a cognitive perspective on understanding and treatment. The Lancet Psychiatry, 3 , 685-692. doi: 10.1016/S2215-0366(16)00066-3

Freeman, D., Loe, B. S., Kingdon, D., Startup, H., Molodynski, A., Rosebrock, L., Brown, P., Sheaves, B., Waite, F., \& Bird, J. C. (2019). The revised Green et al., Paranoid Thoughts Scale (R-GPTS): psychometric properties, severity ranges, and clinical cut-offs. Psychological Medicine, 1-10. doi: 10.1017/S0033291719003155

Freeman, D., Startup, H., Dunn, G., Černis, E., Wingham, G., Pugh, K., Cordwell, J., \& Kingdon, D. (2013). The interaction of affective with psychotic processes: a test of the effects of worrying on working memory, jumping to conclusions, and anomalies of experience in patients with persecutory delusions. Journal of Psychiatric Research, 47 , 1837-1842. doi: 10.1016/j.psychires.2013.06.016

Garety, P. A., Kuipers, E., Fowler, D., Freeman, D., \& Bebbington, P. E. (2001). A cognitive model of the positive symptoms of psychosis. Psychological Medicine, 31, 189-95. doi: 10.1017/s0033291701003312

Hunter, E. C. M., Phillips, M. L., Chalder, T., Sierra, M., \& David, A. S. (2003). Depersonalisation disorder: a cognitivebehavioural conceptualisation. Behaviour Research and Therapy, 41, 1451-1467. doi: 10.1016/S0005-7967(03)00066-4

Hunter, E. C. M., Salkovskis, P. M., \& David, A. S. (2014). Attributions, appraisals and attention for symptoms in depersonalisation disorder. Behaviour Research and Therapy, 53, 20-29. doi: 10.1016/j.brat.2013.11.005

Kennedy, F., Kennerley, H., \& Pearson, D. (eds) (2013). Cognitive Behavioural Approaches to the Understanding and Treatment of Dissociation (pp. 1-16). New York, USA: Routledge.

Laddis, A., \& Dell, P. F. (2012). Dissociation and psychosis in dissociative identity disorder and schizophrenia. Journal of Trauma \& Dissociation, 4, 397-413. doi: 10.1080/15299732.2012.664967

Longden, E., Branitsky, A., Moskowitz, A., Berry, K., Bucci, S., \& Varese, F. (2020). The relationship between dissociation and symptoms of psychosis: a meta-analysis. Schizophrenia Bulletin, sbaa037. doi: 10.1093/schbul/sba037

Lyssenko, L., Schmahl, C., Bockhacker, L., Vonderlin, R., Bohus, M., \& Kleindienst, N. (2018). Dissociation in psychiatric disorders: a meta-analysis of studies using the Dissociative Experiences Scale. American Journal of Psychiatry, 175, 37-46. doi: 10.1176/appi.ajp.2017.17010025

Maher, B. A. (1974). Delusional thinking and perceptual disorder. Journal of Individual Psychology, 30, 98-113.

Qualtrics (2018). [online software]. USA: Provo, Utah. Retrieved from: www.qualtrics.com

R Core Team $(2018,2020)$ R: A Language and Environment for Statistical Computing. R Foundation for Statistical Computing. Retrieved from: http://www.R-project.org/

Renard, S. B., Huntjens, R. J., Lysaker, P. H., Moskowitz, A., Aleman, A., \& Pijnenborg, G. H. (2017). Unique and overlapping symptoms in schizophrenia spectrum and dissociative disorders in relation to models of psychopathology: a systematic review. Schizophrenia Bulletin, 43, 108-121. doi: 10.1093/schbul/sbw063

Revelle, W. (2018, 2019) psych: Procedures for Personality and Psychological Research, Northwestern University, Evanston, Illinois, USA. https://CRAN.R-project.org/package = psych

Ronald, A., Sieradzka, D., Cardno, A. G., Haworth, C. M. A., McGuire, P., \& Freeman, D. (2013). Characterization of psychotic experiences in adolescence using the Specific Psychotic Experiences Questionnaire: findings from a study of 5000 16-year-old twins. Schizophrenia Bulletin, 40, 868-877. doi: 10.1093/schbul/sbt106

Rosseel, Y. (2018, 2019). lavaan: an R Package for Structural Equation Modeling. Journal of Statistical Software, 48, 1-36. http://www.jstatsoft.org/v48/i02/

Samuelson, K. W., Bartel, A., Valadez, R., \& Jordan, J. T. (2017). PTSD symptoms and perception of cognitive problems: the roles of posttraumatic cognitions and trauma coping self-efficacy. Psychological Trauma: Theory, Research, Practice, and Policy, 9, 537-544. doi: 10.1037/tra0000210

Stopa, L., Denton, R., Wingfield, M., \& Newman Taylor, K. (2013). The fear of others: a qualitative analysis of interpersonal threat in social phobia and paranoia. Behavioural and Cognitive Psychotherapy, 41, 188-209. doi: 10.1017/ S1352465812000422

van Buuren, S., \& Groothuis-Oudshoorn, K. (2011, 2020). mice: Multivariate Imputation by Chained Equations in R. Journal of Statistical Software, 45, 1-67. https://www.jstatsoft.org/v45/i03/ 


\section{Appendix: The Cognitive Appraisals of Dissociation in Psychosis (CAD-P) measure}

Please rate how often you think the following when you are feeling strange, disconnected, unreal or 'dissociated'. Please note that this should NOT be whilst under the influence of drugs, alcohol or legal highs.

\begin{tabular}{|c|c|c|c|c|c|c|}
\hline & & Never & Rarely & Sometimes & Often & Always \\
\hline 1 & I can't trust my own mind. & 0 & 1 & 2 & 3 & 4 \\
\hline 2 & Someone has done something to me. & 0 & 1 & 2 & 3 & 4 \\
\hline 3 & Something is terribly wrong. & 0 & 1 & 2 & 3 & 4 \\
\hline 4 & I'm losing my mind. & 0 & 1 & 2 & 3 & 4 \\
\hline 5 & I'm not really 'me'. & 0 & 1 & 2 & 3 & 4 \\
\hline 6 & I am all alone. & 0 & 1 & 2 & 3 & 4 \\
\hline 7 & I don't look right to other people right now. & 0 & 1 & 2 & 3 & 4 \\
\hline 8 & I must be sick. & 0 & 1 & 2 & 3 & 4 \\
\hline 9 & I'm not in the same world as everyone else. & 0 & 1 & 2 & 3 & 4 \\
\hline 10 & This is because I am evil. & 0 & 1 & 2 & 3 & 4 \\
\hline 11 & Now I won't be able to do the things I wanted. & 0 & 1 & 2 & 3 & 4 \\
\hline 12 & It's not me in control right now. & 0 & 1 & 2 & 3 & 4 \\
\hline 13 & This might last forever. & 0 & 1 & 2 & 3 & 4 \\
\hline
\end{tabular}

Scoring: sum score of all items.

Note that: raw scores cannot distinguish between many appraisals occurring infrequently, and a small number of appraisals experienced very frequently. In clinical contexts, therefore, further interpretation of responses may be required.

Cite this article: Černis E, Bird JC, Molodynski A, Ehlers A, and Freeman D (2021). Cognitive appraisals of dissociation in psychosis: a new brief measure. Behavioural and Cognitive Psychotherapy 49, 472-484. https://doi.org/10.1017/ S1352465820000958 